\title{
Frontières
}

\section{Nouvelles parutions - Études sur la mort, Serge Gariépy, bibliothécaire de référence, Bibliothèque centrale, UQAM.}

Volume 21, numéro 1, automne 2008

Prévenir le suicide

URI : https://id.erudit.org/iderudit/037894ar

DOI : https://doi.org/10.7202/037894ar

Aller au sommaire du numéro

Éditeur(s)

Université du Québec à Montréal

ISSN

1180-3479 (imprimé)

1916-0976 (numérique)

Découvrir la revue

Citer ce document

(2008). Nouvelles parutions - Études sur la mort, Serge Gariépy, bibliothécaire de référence, Bibliothèque centrale, UQAM. Frontières, 21(1), 148-148.

https://doi.org/10.7202/037894ar d'utilisation que vous pouvez consulter en ligne. 
NEVES, F. S., MALLOY-DINIZ, L. F. et H. CORREA (2008). "Suicidal behavior in bipolar disorder: What is the influence of psychiatric comorbidities? ", Journal of Clinical of Psychiatry, in press.

SHER, L., OQUENDO, M. A., RICHARDSONVEJLGAARD, R., MAKHIJA, N. M., POSNER, K., MANN, J. J. et B. H. STANLEY (2009). «Effect of acute alcohol use on the lethality of suicide attempts in patients with mood disorders ", Journal of Psychiatric Research, in press.

TIDEMALM, D., LANGSTROM, N., LICHTENSTEIN, P. et B. RUNESON (2008). « Risk of suicide after suicide attempt according to coexisting psychiatric disorder: Swedish cohort study with long term follow-up ", BMJ, 337, p. a2205.

\section{AUTRES FACTEURS DE RISQUE}

BAUD, P., PERROUD, N., COURTET, P., JAUSSENT, I., RELECOM, C., JOLLANT, F. et A. MALAFOSSE (2009). «Modulation of anger control in suicide attempters by TPH-1 ", Genes, Brain and Behavior, vol. 8, n० 1, p. 97-100.

BJORK PETERSEN, C., GRONBAEK, M. N., BUSSEY RASK, M., NIELSEN, B. et A. SOGAARD NIELSEN (2008). "Suicidal behaviour among alcoholdependent Danes attending outpatient treatment ", Nordic Journal of Psychiatry, p. 1-8.

FAZAA, N. et S. PAGE (2009). «Personality style and impulsivity as determinants of suicidal subgroups ", Archives of Suicide Research, vol. 13, $n^{\circ} 1$, p. 31-45.

O'DONNELL, M. L., CREAMER, M., ELLIOTT, P., BRYANT, R., MCFARLANE, A. et D. SILOVE (2009). «Prior trauma and psychiatric history as risk factors for intentional and unintentional injury in Australia ", The Journal of Trauma, vol. 66, $n^{\circ} 2$, p. 470-476.

PEDERSEN, W. et T. VON SOEST (2009). «Smoking, nicotine dependence and mental health among young adults: a 13-year population-based longitudinal study", Addiction, vol. 104, n 1, p. 129 137.

RIALA, K., TAANILA, A., HAKKO, H. et P. RASANEN (2009). "Longitudinal smoking habits as risk factors for early-onset and repetitive suicide attempts: the Northern Finland 1966 Birth Cohort study ", Annals of Epidemiology, in press.

SORENSEN, H. J., MORTENSEN, E. L., WANG, A. G., JUEL, K., SILVERTON, L. et S. A. MEDNICK (2009). "Suicide and mental illness in parents and risk of suicide in offspring: A birth cohort study", Social Psychiatry and Psychiatric Epidemiology, in press.

STIRN, A. et A. HINZ (2008). «Tattoos, body piercings, and self-injury: is there a connection? Investigations on a core group of participants practicing body modification ", Psychotherapy Research, vol. 18, n³, p. 326-333.

WYDER, M., WARD, P. et D. DE LEO (2009). «Separation as a suicide risk factor ", Journal of Affective Disorders, in press.

\section{MÉDIA}

FU, K. W. et P. S. YIP (2008). "Changes in reporting of suicide news after the promotion of the WHO media recommendations", Suicide and LifeThreatening Behavior, vol. 38, n 5, p. 631-636.

\section{NOUVELLES PARUTIONS - ÉTUDES SUR LA MORT}

Serge Gariépy, bibliothécaire de référence, Bibliothèque centrale, UQAM.

BEAUTHÉAC-BOUCHART, Nadine (2008). Hommes et femmes face au deuil: regards croisés sur le chagrin, Paris, Albin Michel.

BÉLAND, Jean-Pierre (2008). Mourir dans la dignité? Soins palliatifs ou suicide assisté, un choix de société, Québec, Presses de I'Université Laval.

BOISVENU, Pierre-Hugues (2008). Survivre à l'innommable et reprendre le pouvoir sur sa vie, Montréal, Éditions de l'Homme.

BROHM, Jean-Marie (2008). Figures de la mort: perspectives critiques, Paris, Beauchesne.

CARLOS, France (2008). Le deuil animalier, SaintConstant, Broquet.

CROS, Michèle (2008). Déjouer la mort en Afrique: or, orphelins, fantômes, trophées et fétiches, Paris, L'Harmattan.

JACQUES, Josée (2008). Le milieu funéraire démystifié, Outremont, Quebecor.

KEIRSE, Manu (2008). Faire son deuil, vivre un chagrin: un guide pour les proches et les professionnels, Bruxelles, De Boeck.

KENTISH-BARNES, Nancy (2008). Mourir à I'hôpital: décisions de fin de vie en réanimation, Paris, Seuil.

LAFONTAINE, Céline (2008). La société post-mortelle: la mort, l'individu et le lien social à l'ère des technosciences, Paris, Seuil.
LAPLANTE, Francine et Michel LEBOEUF (2008). Jusqu'au bout de ta courte vie: accompagner son enfant malade vers la mort et y survivre, Montréal, Éditions La Presse.

LÉGER, Pierre (2008). La mort et l'au-delà, Montréal, Novalis.

LEGROS, Patrick et Carine HERBÉ (2006). La mort au quotidien: contribution à une sociologie de I'imaginaire de la mort et du deuil, RamonvilleSaint-Agne, Erès.

MAUGEIN, Patrick (2006). Ouï-dire sur la mort, Paris, J.-C. Gawsewitch.

MONBOURQUETTE, Jean (2008). La mort, ça s'attrape?, Ottawa, Novalis.

MYTTENAERE, Chantal (2007). Panser le deuil, Paris, Éditions de l'Hèbe.

RAY, Ruth E. (2008). Endnotes: An Intimate Look at the End of Life, New York, Columbia University Press.

ROGERS, J. Earl (2007). The Art of Grief. The Use of Expressive Arts in a Grief Support Group, New York, Routledge.

THIEL, Marie-Jo (2008). Les rites autour du mourir, Strasbourg, Presses universitaires de Strasbourg.

WEE, Bee et Nic Hughes (2007). Education in Palliative Care: Building a Culture of Learning, Oxford, Oxford University Press.

WEINSTEIN, Jeremy (2008). Working with Loss, Death and Bereavement: A Guide for Social Workers, Thousand Oaks (CA), Sage Publications.

ZECH, Emmanuelle (2007). Psychologie du deuil: impact et processus d'adaptation au décès d'un proche, Wavre, Belgique, Mardaga.

\section{APPEL D'ARTICLES}

\author{
Sur les thèmes suivants : \\ Résilience et deuil \\ Enquêtes sur le cadavre
}

Avant de soumettre votre article, veuillez consulter le protocole de rédaction de la revue Frontières disponible à l'adresse suivante: www.frontieres.uqam.ca/pdf/protocole-de-redaction.pdf

Faites-nous ensuite parvenir votre texte à : frontieres@uqam.ca

Les articles soumis seront évalués de façon anonyme par un comité scientifique externe. 